\title{
Research on inversion technique of equivalent excitations of flexible panel structures
}

\author{
Xuetao Weng ${ }^{1}$, Rui Huo ${ }^{2}$, Weike Wang ${ }^{2}$ a and Zhidong Wang ${ }^{2}$ \\ ${ }^{1}$ Key Laboratory of Shipping Noise and Vibration, Naval University of engineering, Wu'han 430033, China \\ ${ }^{2}$ School of Mechanical Engineering, Shandong University, Ji'nan250061, China
}

\begin{abstract}
Detection of sonar platform excitations has been a formidable obstacle towards the estimation and evaluation of mechanical self-noise in the sonar cavity. In view of the complexity of transmission of excitations from mechanical vibration equipments to sonar platform, an inversion technique, i.e., indirectly estimating the outside excitations by means of measurement of vibratory responses, would be the most applicable and feasible. An equivalent excitation method is proposed in this paper, by the hypothesis that responses resulted from equivalent excitations would be equal to that from actual excitations, to deal with the excitation inverse problem in situations where there is no knowing about the location and distribution of dynamic excitation forces acting on structures. Influential factors in estimation of equivalent excitations, including determination of master modals, number and arrangement of observation points and equivalent excitation points, are well reasoned based on modal theory of panel structures, with analytical analysis method. And it could be concluded that the equivalent excitation inversion would be a practical method for evaluation of environmental excitations.
\end{abstract}

Keywords: inversion of excitation spectrum; equivalent excitation; plate and shell structures; master modals.

\section{Introduction}

In practical engineering problems, direct excitation measurement is difficult even it is difficult to realize, and according to the structural vibration response to the incentive for indirect estimation method has been widely used. As a result, forming a dynamic load identification technology field. In the ship shore based maintenance, by measuring the installation based on vibration response of the source equipment incentive levels were assessed, or using the measured the sonar array cavity wall vibration response without water filling estimated that the external mechanical excitation as the assessment reference for evaluation of mechanical self-noise of sonar. The difficulty of this kind of problem lies in the fact that only a finite number of measuring points can be chosen to measure the vibration response, and the exact reconstruction of the continuous system excitation needs to identify the infinite unknown quantity. Therefore, how to improve the accuracy of estimation, simplify the test and estimation process is a focus of the research work.

In this paper, based on the theory of vibration mode of flexible panel structures, the general method of estimating equivalent excitation spectrum based on finite vibration response data is theoretically analyzed and demonstrated. Taking into account the complexity of the practical test,

a Corresponding author : 449200779@qq.com

(C) 2016. The authors - Published by Atlantis Press 
using the Navier solution of rectangular thin plate with simply supported edges of equivalent 错误! 未 定义书签。 excitation estimation experiment scheme design of the reliability of the main factors were studied, including mainly affect the mode selection, measuring point number and setpoint cloth Bureau, equivalent excitation point number and position setting and so on.

\section{Theory for equivalent excitation inversion of flexible panel structures}

Differential equation of vibration for flexible panel

$$
\nabla^{4} w(\sigma, \mathrm{t})+\frac{\rho h}{D} \frac{\partial^{2} w(\sigma, \mathrm{t})}{\partial t^{2}}=\frac{f(\sigma, \mathrm{t})}{D}
$$

Assuming that excitation $\mathrm{f}(\sigma, \mathrm{t})$ is harmonic, the vibration response $\mathrm{w}(\sigma, \mathrm{t})$ is harmonic.

$$
\begin{gathered}
\mathrm{w}(\sigma, \mathrm{t})=\mathrm{W}(\sigma, \omega) \mathrm{e}^{\mathrm{j} \omega \mathrm{t}}=\sum_{i=1}^{\infty} \frac{\Psi_{i}(\sigma) Q_{i} \mathrm{e}^{\mathrm{j} \omega t}}{M_{i}\left(\omega_{i}^{2}-\omega^{2}\right)}=\sum_{i=1}^{\infty} \xi_{i}(\omega) \Psi_{i}(\sigma) \mathrm{e}^{\mathrm{j} \omega t} \\
Q_{i}=\iint_{A} F(\sigma) \Psi_{i}(\sigma) \mathrm{d} \sigma
\end{gathered}
$$

It selects the $\mathrm{N}$ order modal of significant impact factor to synthesize $w(\sigma, t)$, that is

$$
W(\sigma, \omega)=\sum_{i=\chi}^{\chi+N-1} \xi_{i}(\omega) \Psi_{i}(\sigma)+\varepsilon
$$

Equivalent excitation estimation problem is mainly used for low and medium frequency, so in the Eq. (2), let $\chi=1$, and selecting $\sigma_{1}, \sigma_{2}, \ldots, \sigma_{M}$ as the observation points to obtain observational value $\mathrm{W}=\left[\mathrm{W}\left(\sigma_{1}, \omega\right), \mathrm{W}\left(\sigma_{2}, \omega\right), \ldots, \mathrm{W}\left(\sigma_{\mathrm{M}}, \omega\right)\right]^{\mathrm{T}}$, the observation matrix equation can be obtained

$$
\boldsymbol{W}=\left[\begin{array}{cccc}
\Psi_{1}\left(\sigma_{1}\right) & \Psi_{2}\left(\sigma_{1}\right) & \ldots & \Psi_{N}\left(\sigma_{1}\right) \\
\Psi_{1}\left(\sigma_{2}\right) & \Psi_{2}\left(\sigma_{2}\right) & \ldots & \Psi_{N}\left(\sigma_{2}\right) \\
\vdots & \vdots & \ddots & \vdots \\
\Psi_{1}\left(\sigma_{M}\right) & \Psi_{2}\left(\sigma_{M}\right) & \ldots & \Psi_{N}\left(\sigma_{M}\right)
\end{array}\right]\left[\begin{array}{c}
\xi_{1} \\
\xi_{2} \\
\vdots \\
\xi_{N}
\end{array}\right]+\left[\begin{array}{c}
\varepsilon_{1} \\
\varepsilon_{2} \\
\vdots \\
\varepsilon_{M}
\end{array}\right]=\boldsymbol{\Psi}_{\xi}+\boldsymbol{\varepsilon}
$$

Applying the least square estimation principle, the least $\boldsymbol{\xi}$ making residual $\boldsymbol{\varepsilon}^{\mathrm{T}} \boldsymbol{\varepsilon}$ is

$$
\xi=\left(\Psi^{T} \Psi\right)^{-1} \cdot \Psi^{T} W
$$

By Eq. (2) it obtained the generalized excitation of the main effect modal

$$
\boldsymbol{Q}=\boldsymbol{M}\left(\boldsymbol{\Omega}^{2}-\omega^{2} \boldsymbol{I}_{N}\right) \boldsymbol{\xi}
$$

In the formula, $I_{N}$ expresses the $N$ order unit matrix, $M=\operatorname{diag}\left[M_{1}, M_{2}, \ldots, M_{N}\right], \Omega=\operatorname{diag}\left[\omega_{1}, \omega_{2}, \ldots\right.$, $\left.\omega_{\mathrm{N}}\right], \mathrm{Q}=\left[\mathrm{Q}_{1}, \mathrm{Q}_{2}, \ldots, \mathrm{Q}_{\mathrm{N}}\right]^{\mathrm{T}}$.

Integrating the Eq.(1) (6), by solving the linear equations, the equivalent excitation $\mathrm{F}_{\mathrm{eq}}$ has the same effect as the actual excitation $\mathrm{F}(\sigma)$

$$
\boldsymbol{Q}=\left[\begin{array}{cccc}
\Psi_{1}\left(\sigma_{\text {eq }}\right) & \Psi_{1}\left(\sigma_{\text {eq }}\right) & \ldots & \Psi_{1}\left(\sigma_{\text {equ }}\right) \\
\Psi_{2}\left(\sigma_{\text {eq1 }}\right) & \Psi_{2}\left(\sigma_{\text {eq }}\right) & \cdots & \Psi_{2}\left(\sigma_{\text {eqL }}\right) \\
\vdots & \vdots & \ddots & \vdots \\
\Psi_{N}\left(\sigma_{\text {eq1 }}\right) & \Psi_{N}\left(\sigma_{\text {eq } 2}\right) & \cdots & \Psi_{N}\left(\sigma_{\text {eq }}\right)
\end{array}\right] \boldsymbol{F}_{\text {eq }}=\Psi_{\text {eq }} \boldsymbol{F}_{\text {eq }}
$$

The above equivalent excitation method is based on the assumption that the position of the 
excitation effect is completely unknown. In the case of a certain $F(\sigma)$ distribution of information, by the matrix pseudo inverse theory a minimum square sum of the equivalent excitation solution is as follows

$$
\boldsymbol{F}_{\text {eq }}=\left(\boldsymbol{\Psi}_{\text {eq }}\right)^{\mathrm{T}}\left[\boldsymbol{\Psi}_{\text {eq }} \cdot\left(\boldsymbol{\Psi}_{\text {eq }}\right)^{\mathrm{T}}\right]^{-1} \boldsymbol{M}\left(\boldsymbol{\Omega}^{2}-\omega^{2} \boldsymbol{I}_{N}\right) \cdot\left(\boldsymbol{\Psi}^{\mathrm{T}} \boldsymbol{\Psi}\right)^{-1} \cdot \boldsymbol{\Psi}^{\mathrm{T}} \boldsymbol{W}
$$

\section{Analysis of theoretical accuracy and influence factors of equivalent excitation estimation}

\subsection{Determination of the main impact modal}

The rectangular thin plate with simply supported edges based on the Navier method is considered, and its natural frequency, modal function and modal mass are respective

$$
\begin{gathered}
\omega_{i}=\pi^{2} \sqrt{\frac{D}{\rho h}\left[\left(\frac{m_{i}}{L_{X}}\right)^{2}+\left(\frac{n_{i}}{L_{Y}}\right)^{2}\right]} \\
\Psi_{i}(\sigma)=\sin \left(\frac{m_{i} \pi x}{L_{X}}\right) \sin \left(\frac{n_{i} \pi y}{L_{Y}}\right) \\
\mathrm{M}_{\mathrm{i}}=\rho \mathrm{hL}_{\mathrm{X}} \mathrm{L}_{\mathrm{Y}} / 4
\end{gathered}
$$

In the formula, $\mathrm{i}=1,2, \ldots,\left(\mathrm{m}_{\mathrm{i}}, \mathrm{n}_{\mathrm{i}}\right) \in \mathrm{N}^{2}$, and let $\omega_{1}<\omega_{2}<\ldots, L_{X}, L_{Y}$ are the length of rectangular plate.

The rectangular plate is divided into grid and the grid node number, as shown in Fig. 1. Assuming that the length of the rectangular plate $L_{X}=0.6 \mathrm{~m}, L_{Y}=0.4 \mathrm{~m}$, the thickness $h=0.005 \mathrm{~m}$, material is $45 \#$ steel. Some nodes were randomly selected on board applied to harmonic excitation. Respectively in the third, 23th and 27th nodes which applied unit harmonic force of $500 \mathrm{~Hz}$ frequency calculated modal impact factor before the 25th order.

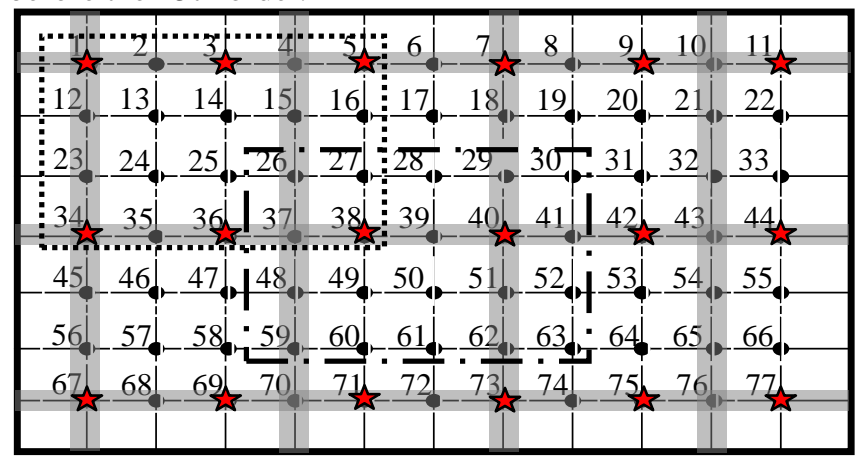

Figure 1. Grid node number and arrangement of observation points/equivalent excitation points of rectangular plate

It is assumed that the vibration response of the 1 77 node in the plate is obtained by excitation frequencies in the vicinity of the fifth, 10th, 15th, and 20th order modals and the modal impact factors (amplitude $|W(\sigma)|$ ), and compares the results in Fig. 2. 


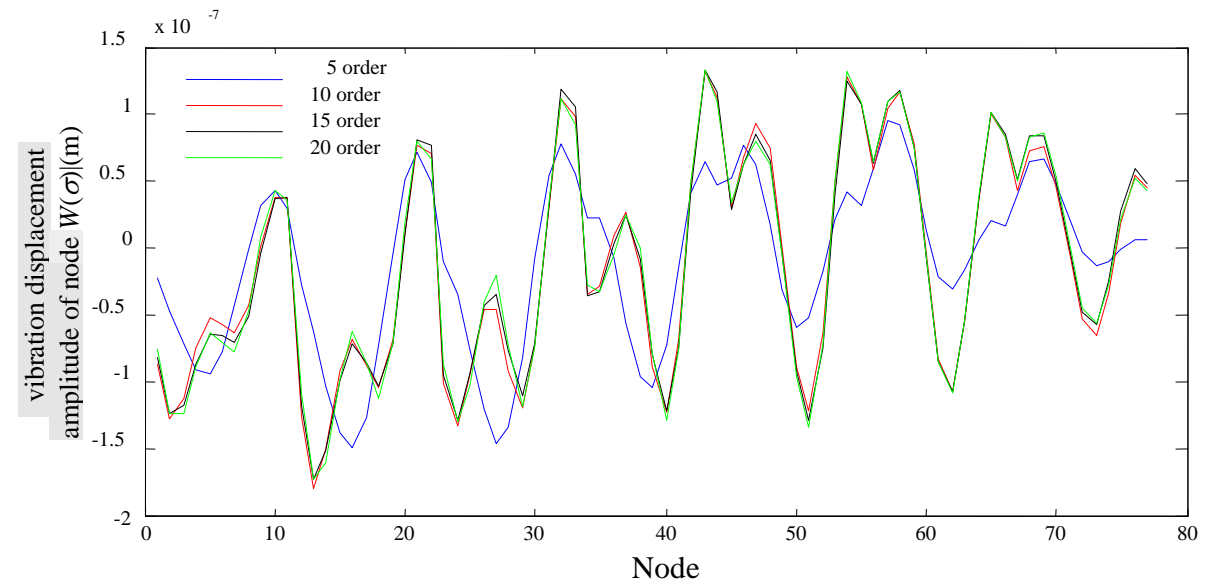

Figure 2. Comparison of the results of the displacement response of the stacking synthetic node with modal superposition of different orders

\subsection{The number of observation points and the selection of layout}

Considering the feasibility of a large number of test points in the actual operation and the testing workload, we should seek to obtain more accurate and reliable calculation results with less number of measuring points and testing work.

On the figure 1 rectangular thin plate, if the 10th order modal in the vicinity of the excitation frequency is selected as the main modal, and the number of the observation points $M \geq 10$ should be guaranteed. In order to study the number of observation points and the effect of estimation accuracy of the equivalent excitation on the choice of site layout, consider the following several different observation points selection scheme, (1) Take all 77 nodes as the observation point in Figure 1, (2) Take 49 nodes in the shadow part as the observation point in Figure 1, (3) Take 12 nodes in the shadow of overlap as the observation point in Figure 1, (4) Take 20 nodes contained in the point line box as the observation point in Figure 1, (5) Take 20 nodes contained in the dotted box as the observation point in Figure 1.

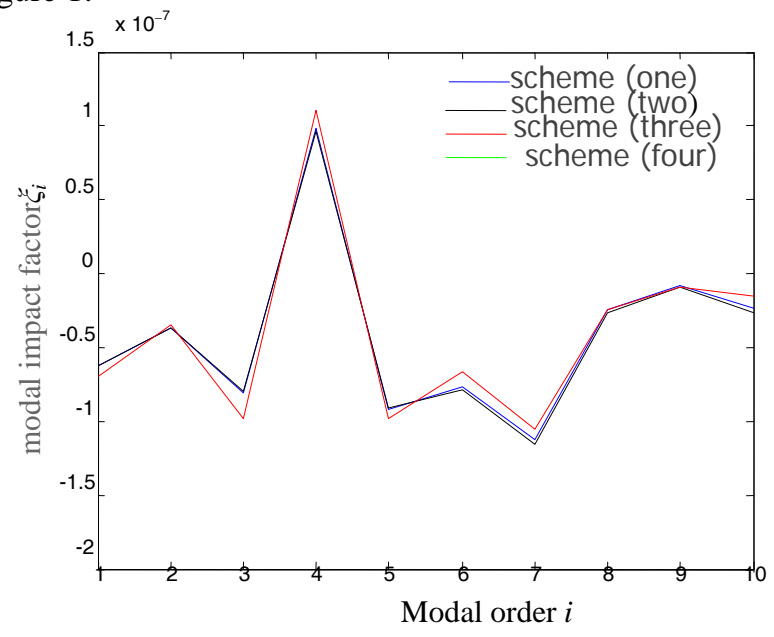

Figure 3. Comparison of modal impact factors of different observation points layout schemes

Applying a $500 \mathrm{~Hz}$ unit harmonic concentrated force on the rectangular plate (the 27th node). The vibration displacement of 1 77 number in the plate calculated by the first 25 order modal superposition is considered as a response observation. Estimating the modal impact factor $\xi$ by the Eq. 
(6). Fig. 3 is a comparison of the results of the estimation of the first 10 modal impact factors under the 5 observation points. That scheme (one) does not have much difference between the results and the theoretical values.

\subsection{The setting of equivalent excitation points}

The setting of equivalent excitation function point can be speculated by the actual incentive situation, or if it does not care about the exact location of the actual excitation, then it can freely set the equivalent incentive points. Because there is no unique equivalent incentive point setting scheme, the reliability of equivalent excitation estimation is evaluated by the approximate degree of the vibration response of the structure under the effect of equivalent excitation and the effect of the actual excitation.

\section{Conclusion}

This paper based on response similar to the principle of equivalent excitation method as a kind of ambient excitation inversion method to evaluate the reliability and practicability of theory analysis and demonstration. When the excitation position cannot be determined, the equivalent excitation spectrum can be used for the inversion effect on actual excitation to simulate and analyze. If the excitation position is known, the identification of the excitation size can be realized by the inversion of the equivalent excitation spectrum.

In addition to the measurement error of the vibration response, the precision of the equivalent excitation estimation is affected by the main factors such as the mode selection, the number and the arrangement of the measuring points.

\section{References}

1. Yu.Ming. Mao and Jian.Feng. Lin and Jing.Hua. Liu and Wen.Bin. Di, Review of research on dynamic load inversion analysis technology [J]. Journal of dynamics and control, 2014, 12 (2):97-104.

2. Yin.Yin. $\mathrm{Hu}$ and Zhi.Jun. Shuai and Wan.You. Li and Zhi.Gang. Liu, Research status of equipment load identification and excitation source characteristics [J]. noise and vibration control, 2011, 31 (4):1-5.

3. Cui.Ping. Liu, The analysis of the mechanical self-noise propagation in the acoustic field of sonar [D]. Shandong University master's thesis [D]. Shandong University master's thesis, 2015.

4. J. Sanchez and H. Benaroya, Review of force reconstruction techniques[J]. Journal of Sound and Vibration, 2014, 333: 2999-3018.

5. A.N. Thite and D.J. Thompson, Selection of response measurement locations to improve inverse force determination[J]. Applied Acoustics, 2006, 67: 797-818. 